\title{
SPITZER IRS SPECTRA OF OPTICALLY FAINT INFRARED SOURCES WITH WEAK SPECTRAL FEATURES
}

\author{
D. W. Weedman, ${ }^{1}$ B. T. Solfer, ${ }^{2,3}$ Lei Hao, ${ }^{1}$ J. L. Higdon, ${ }^{1}$ S. J. U. Higdon, ${ }^{1}$ J. R. Houck, ${ }^{1}$ E. Le Floc'h, ${ }^{4}$ \\ M. J. I. Brown, ${ }^{5,6}$ A. Dey, ${ }^{6}$ B. T. Jannuzi, ${ }^{6}$ M. Rieke, ${ }^{4}$ V. Desai, ${ }^{3}$ C. Bian, ${ }^{3}$ D. Thompson, ${ }^{3}$ \\ L. Armus, ${ }^{2}$ H. Teplitz, ${ }^{2}$ P. Eisenhardt, ${ }^{7}$ and S. P. Willner ${ }^{8}$ \\ Received 2006 May 16; accepted 2006 June 28
}

\begin{abstract}
Spectra have been obtained with the low-resolution modules of the Infrared Spectrograph (IRS) on the Spitzer Space Telescope (Spitzer) for 58 sources having $f_{\nu}(24 \mu \mathrm{m})>0.75 \mathrm{mJy}$. Sources were chosen from a survey of $8.2 \mathrm{deg}^{2}$ within the NOAO Deep Wide-Field Survey region in Boötes (NDWFS) using the Multiband Imaging Photometer (MIPS) on Spitzer. Most sources are optically very faint ( $I>24$ mag). Redshifts have previously been determined for 34 sources, based primarily on the presence of a deep $9.7 \mu \mathrm{m}$ silicate absorption feature, with a median $z$ of 2.2. Spectra are presented for the remaining 24 sources for which we were previously unable to determine a confident redshift because the IRS spectra show no strong features. Optical photometry from the NDWFS and infrared photometry with MIPS and the Infrared Array Camera on Spitzer (IRAC) are given, with $K$ photometry from the Keck I telescope for some objects. The sources without strong spectral features have overall spectral energy distributions (SEDs) and distributions among optical and infrared fluxes that are similar to those for the sources with strong absorption features. Nine of the 24 sources are found to have feasible redshift determinations based on fits of a weak silicate absorption feature. Results confirm that the " $1 \mathrm{mJy}$ " population of $24 \mu \mathrm{m}$ Spitzer sources that are optically faint is dominated by dusty sources with spectroscopic indicators of an obscured AGN rather than a starburst. There remain 14 of the 58 sources observed in Boötes for which no redshift could be estimated, and 5 of these sources are invisible at all optical wavelengths.
\end{abstract}

Subject headings: dust, extinction — galaxies: active — galaxies: high-redshift — galaxies: starburst — infrared: galaxies

\section{INTRODUCTION}

Imaging surveys at infrared wavelengths with the Spitzer Space Telescope (Spitzer) have the potential to reveal populations of sources that were previously unknown. In particular, surveys with the Multiband Imaging Photometer for Spitzer (MIPS; Rieke et al. 2004) at $24 \mu \mathrm{m}$ should reveal sources that are luminous because of emission from dust and that may contain sufficient dust to make them very faint or invisible optically. The Infrared Spectrograph on Spitzer (IRS; ${ }^{9}$ Houck et al. 2004a) is sufficiently sensitive to obtain low-resolution spectra of these MIPS sources at flux density levels of $f_{\nu}(24 \mu \mathrm{m})<1 \mathrm{mJy}$.

As part of initial efforts to characterize this " $1 \mathrm{mJy}$ " population, we surveyed $8.2 \mathrm{deg}^{2}$ within the Boötes field of the NOAO Deep Wide-Field Survey (NDWFS; Jannuzi \& Dey 1999) with MIPS to produce a catalog of mid-infrared sources. The MIPS data were obtained with an effective integration time at $24 \mu \mathrm{m}$ of $\sim 90 \mathrm{~s}$ per sky pixel, reaching a $5 \sigma$ detection limit of $\sim 0.3 \mathrm{mJy}$ for unre-

\footnotetext{
1 Astronomy Department, Cornell University, Ithaca, NY 14853; dweedman@ astro.cornell.edu.

2 Spitzer Science Center, California Institute of Technology, 220-6, Pasadena, CA 91125.

3 Division of Physics, Mathematics and Astronomy, 320-47, California Institute of Technology, Pasadena, CA 91125.

4 Steward Observatory, University of Arizona, Tucson, AZ 85721.

5 Department of Astrophysical Sciences, Princeton University, Peyton Hall, Princeton, NJ 08544-1001.

${ }^{6}$ National Optical Astronomy Observatory, Tucson, AZ 85726.

7 Jet Propulsion Laboratory, 4800 Oak Grove Drive, 169-327, Pasadena, CA 91109.

8 Smithsonian Astrophysical Observatory, 60 Garden Street, Cambridge, MA 02138.

9 The IRS was a collaborative venture between Cornell University and Ball Aerospace Corporation funded by NASA through the Jet Propulsion Laboratory and the Ames Research Center.
}

solved sources. This field was chosen because the deep and wellcalibrated optical imagery in the $B_{W}, R$, and $I$ bands makes possible the identification of infrared sources with very faint optical counterparts and allows confident selection of infrared sources lacking optical counterparts to very deep optical limits.

We then selected for subsequent spectroscopic observations with Spitzer those sources that are the faintest optically (typically $I>24)$ while also bright enough $(>0.75 \mathrm{mJy}$ at $24 \mu \mathrm{m})$ for spectroscopy with the IRS within integration times of $\sim 1 \mathrm{hr}$. Other than the mid-infrared flux limit, the only selection criterion was optical faintness, because we were primarily interested in understanding sources that would not have been identified in previous optical studies. Our first results reported the discovery of a significant population of optically obscured, high-redshift sources (Houck et al. 2005; hereafter H05). To date, we have observed 58 Boötes sources with the IRS. Continuum was detected in all 58 objects observed, confirming that all mid-infrared sources are real, even when they have no optical counterparts, and also demonstrating that the MIPS-derived positional uncertainties are less than $\sim 0$ ".5 rms. Redshifts for 17 sources determined by fitting templates of known local objects were reported in $\mathrm{H} 05$; an additional 17 sources with redshifts determined include 14 in S. J. U. Higdon et al. (2006, in preparation), two in Desai et al. (2006), and one in Dey et al. (2005). The most important result is that these sources are generally at high redshift. Of the 34 sources with redshifts, the median redshift $(z)$ is 2.2 . The spectra of these sources with redshifts are dominated by strong silicate absorption centered at rest frame $9.7 \mu \mathrm{m}$. Only two sources are best fit by strong polycyclic aromatic hydrocarbon (PAH) emission features not requiring silicate absorption. There remain 24 spectra (the "no-z" sample) that we have observed with the IRS but for which redshifts have not yet been reported because there are no strong spectral features. We discuss these sources in the present paper. 
All Boötes sources with $1.9<z<2.7$ from IRS redshifts have measurable redshifts because of the deep silicate absorption feature centered at rest frame $9.7 \mu \mathrm{m}$. Because of the accessible wavelength range of the IRS, redshifts beyond about 2.8 could not be measured, since this absorption feature would be longward of the IRS wavelength limit. This allows two possible interpretations of the 24 sources for which we have not been able to derive redshifts: either they are a category of sources at redshifts $z \lesssim 2.8$ having weak spectral features, or they are at $z \gtrsim 2.8$. It is crucial to know which conclusion is more representative of the no$z$ sample. If features are weak, that is important for interpreting the nature of the sources and for understanding why they are optically faint. If the no- $z$ sources are at higher redshifts than for the measured sources, this would be evidence for an obscured population at even higher redshifts and luminosities than the sources already identified.

Because this population of optically faint infrared sources would not have been selected using criteria available before Spitzer, it is necessary to consider whether all of the targets do indeed represent a distant, extragalactic population, as we have assumed. Our initial selection ruled out solar system objects by verifying that the infrared source showed no proper motion between the two epochs of the MIPS observations; the selection at $24 \mu \mathrm{m}$ ensures that any solar system objects would be in the main asteroid belt or closer and hence would have measurable proper motion between Spitzer observations. All sources were also detected at the same positions with the Spitzer Infrared Array Camera (IRAC; Fazio et al. 2004), with observations obtained at a different epoch. Known stellar populations are ruled out because the extreme infrared-to-optical flux ratios imply very cool source temperatures for a blackbody. In addition, we report new $K$-band observations with high spatial resolution that resolve at least 4 of the 24 sources. All indications, therefore, are that these sources are an extragalactic population, but locating them in the universe requires the determination of redshifts.

In this paper, we give all available data for the 24 sources with "featureless" spectra and compare the overall spectral energy distributions for this sample of sources to those sources with previously determined redshifts. We illustrate all of the spectra in order to discuss which ones might have weak features and suggest new redshifts for nine sources based on possible but weak spectral absorption features.

\section{OBSERVATIONS AND DATA REDUCTION}

Our initial selection for spectroscopic targets in the Boötes field was to use the MIPS and optical surveys to inspect all sources within the $8.2 \mathrm{deg}^{2}$ portion of the MIPS survey field overlapping the NDWFS that have $f_{\nu}(24 \mu \mathrm{m})>0.75 \mathrm{mJy}$ and $I \geq 24 \mathrm{mag}$. There are 4273 MIPS $24 \mu \mathrm{m}$ sources brighter than $0 . \overline{7} 5 \mathrm{mJy}$, of which 114 met this optical magnitude criterion. (Magnitudes in the NDWFS Data Release 3 are slightly modified from those in the initial version from which we worked, so these statistics would not be exactly correct using the released version.) There are 65 sources of the 114 having $f_{\nu}(24 \mu \mathrm{m})>1.0 \mathrm{mJy}$ and $I>$ 24 mag. To define a sample based on specific infrared and optical flux limits, we observed 35 of the 65 sources with $f_{\nu}(24 \mu \mathrm{m})>$ $1.0 \mathrm{mJy}$ and $I>24 \mathrm{mag}$. We also included 23 sources with extreme values of infrared-to-optical flux ratio even if they did not meet these specific selection criteria. [In the remainder of this paper, we define the infrared-to-optical flux ratio as IR/opt = $\nu f_{\nu}(24 \mu \mathrm{m}) / \nu f_{\nu}(I)$.] These remaining 23 targets are primarily sources with $f_{\nu}(24 \mu \mathrm{m})<1.0 \mathrm{mJy}$ and $I>24 \mathrm{mag}$, but a few sources with optical magnitudes $I<24$ mag were also included.
Spectroscopic observations were made with the IRS Short-Low module in order 1 only (SL1) and with the Long-Low module in orders 1 and 2 (LL1 and LL2), as described in Houck et al. (2004a). These give low-resolution spectral coverage from $\sim 8$ to $\sim 35 \mu \mathrm{m}$. Sources were normally placed on the slits by offsetting from nearby 2MASS stars; in a few cases with no sufficiently nearby 2 MASS stars, direct pointing without offsets was used successfully.

All images when the source was in one of the two nod positions on each slit were co-added to obtain the source spectrum. The background, which was subtracted for LL1 and LL2, included co-added background images that added both nod positions with the source in the other slit together with the alternative nod position in the same slit, yielding a background observation with 3 times the integration time as for the source, in order to reduce noise in the background. The differenced source-minusbackground image was used for the spectral extraction, giving independent extractions of the spectrum at the two positions on the slit for each LL order. The two independent spectra were compared to reject any highly outlying pixels in either spectrum, and a final mean spectrum was produced. For SL1, there was no separate background observation with the source in the SL2 slit, so background subtraction was done between co-added images of the two nod positions in SL1. Observed images were processed with version 11.0 of the SSC pipeline. Extraction of source spectra was done with the SMART analysis package (Higdon et al. 2004). All spectra discussed in this paper are shown in Figures 1 and 2; displayed spectra have been boxcar smoothed to the approximate resolution of the different IRS modules $(0.2 \mu \mathrm{m}$ for $\mathrm{SL1}, 0.3 \mu \mathrm{m}$ for LL2, and $0.4 \mu \mathrm{m}$ for LL1). It can be seen from comparison of the spectral flux densities with the MIPS fluxes (Figs. 1 and 2) that the extracted spectra typically agree at $24 \mu \mathrm{m}$ to within $10 \%$ of the MIPS flux.

Table 1 summarizes the MIPS and IRS observations and resulting characteristics for the 24 sources, giving the name (with full coordinates) and the MIPS $24 \mu \mathrm{m}$ flux density. Sources are ordered by declination. Even when spectral features cannot be seen, the IRS spectra yield a slope of the continuum. To record this information, the power-law slopes of the continua are determined by using SMART to find the best linear fit to the continuum when displayed in log-log units. On occasion with various IRS spectra, the SL continuum does not perfectly stitch to the LL continuum, and this is attributed primarily to slight mispointing, which can cause significant effects in the SL slit. With spectra having poor signal-to-noise ratios $(\mathrm{S} / \mathrm{Ns})$, poor stitching might not be obvious, so the formal measures of the power-law index listed in Table 1 use only LL spectra, with $\lambda>14 \mu \mathrm{m}$. Fits are cut at $33 \mu \mathrm{m}$ because of the increased noise in the spectra that appears beyond this wavelength. The resulting power-law index for $14 \mu \mathrm{m}<\lambda<33 \mu \mathrm{m}$ is given in Table 1 . We have also combined IRAC fluxes with the MIPS results to determine the slope of the power law that would connect the MIPS $24 \mu \mathrm{m}$ and IRAC $8 \mu \mathrm{m}$ fluxes, as well as the power law connecting IRAC $8 \mu \mathrm{m}$ and $3.6 \mu \mathrm{m}$ fluxes. These slopes are also given in Table 1 .

To quantify the quality of the spectra, we measure the $\mathrm{S} / \mathrm{N}$ of the LL1 spectra. This is done by determining within SMART the best-fit continuum to the LL1 spectrum of either linear, quadratic, or cubic fit and then measuring the rms about this continuum. The $\mathrm{S} / \mathrm{N}$ in Table 1 is the ratio $\mathrm{S} / \mathrm{N}=$ (flux density at wavelength midpoint/rms for full continuum fit). Table 1 also includes any new redshift estimates that can be determined from all of the available data, as are discussed below.

More details of near-infrared fluxes and optical magnitudes of the sources are in Table 2, including the IRAC flux measurements 

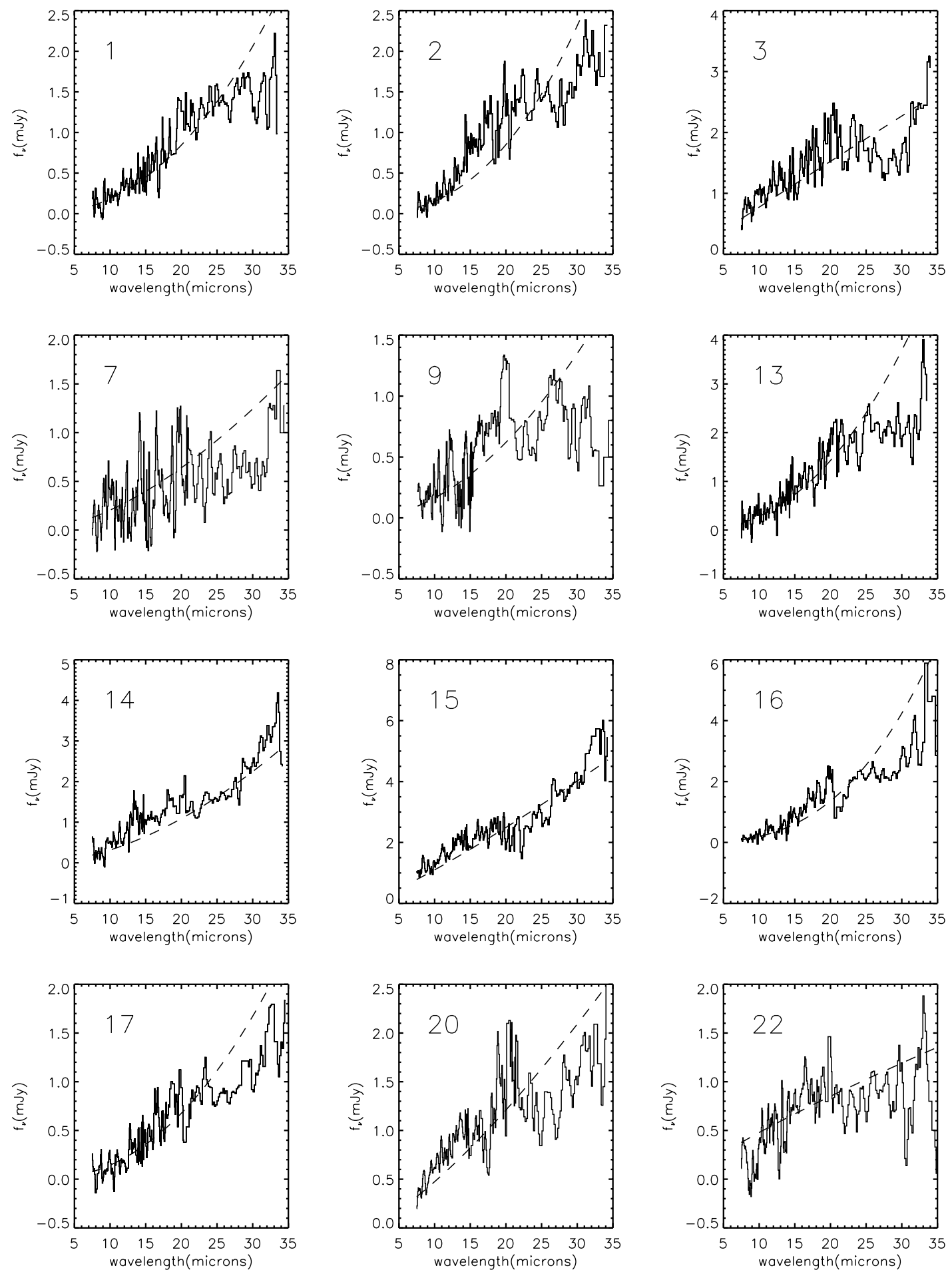

FIG. 1.-Observed spectra of sources in Table 1 that have possible spectral features discussed in the text (histogram). Spectra are smoothed to approximate resolution of individual IRS orders. Dashed curve: Power law that would connect MIPS $f_{\nu}(24 \mu \mathrm{m})$ with the $\operatorname{IRAC} f_{\nu}(8 \mu \mathrm{m})$.

of these sources, observed in Boötes as described by Eisenhardt et al. (2004), along with $K$-band magnitudes.

The $K$-band data for seven objects were obtained on UT 2005 June 19 and 20 with the Near Infrared Camera (NIRC; Matthews $\&$ Soifer 1994) on the Keck I Telescope. The NIRC instrument has a 38 ". 4 field of view and a pixel scale of 0 "' 15 . The seeing was in the range $0.7-0.9$ for all observations. Five objects were observed under photometric conditions on June 20, each for a total exposure time of 20 minutes. Objects 4 and 24 were observed on June 19 under nonphotometric conditions for 16 and 21 minutes, respectively. They were each observed for an additional $200 \mathrm{~s}$ under photometric conditions on June 20.

The individual dithered images for each target were darksubtracted, flat-fielded, masked, aligned, and stacked using the $I_{A A F}{ }^{10}$ NIRCtools package. The data were placed on the Vega magnitude scale using standard stars (Persson et al. 1998) observed

10 IRAF is distributed by the National Optical Astronomy Observatory, which is operated by the Association of Universities for Research in Astronomy, Inc., under cooperative agreement with the National Science Foundation. 

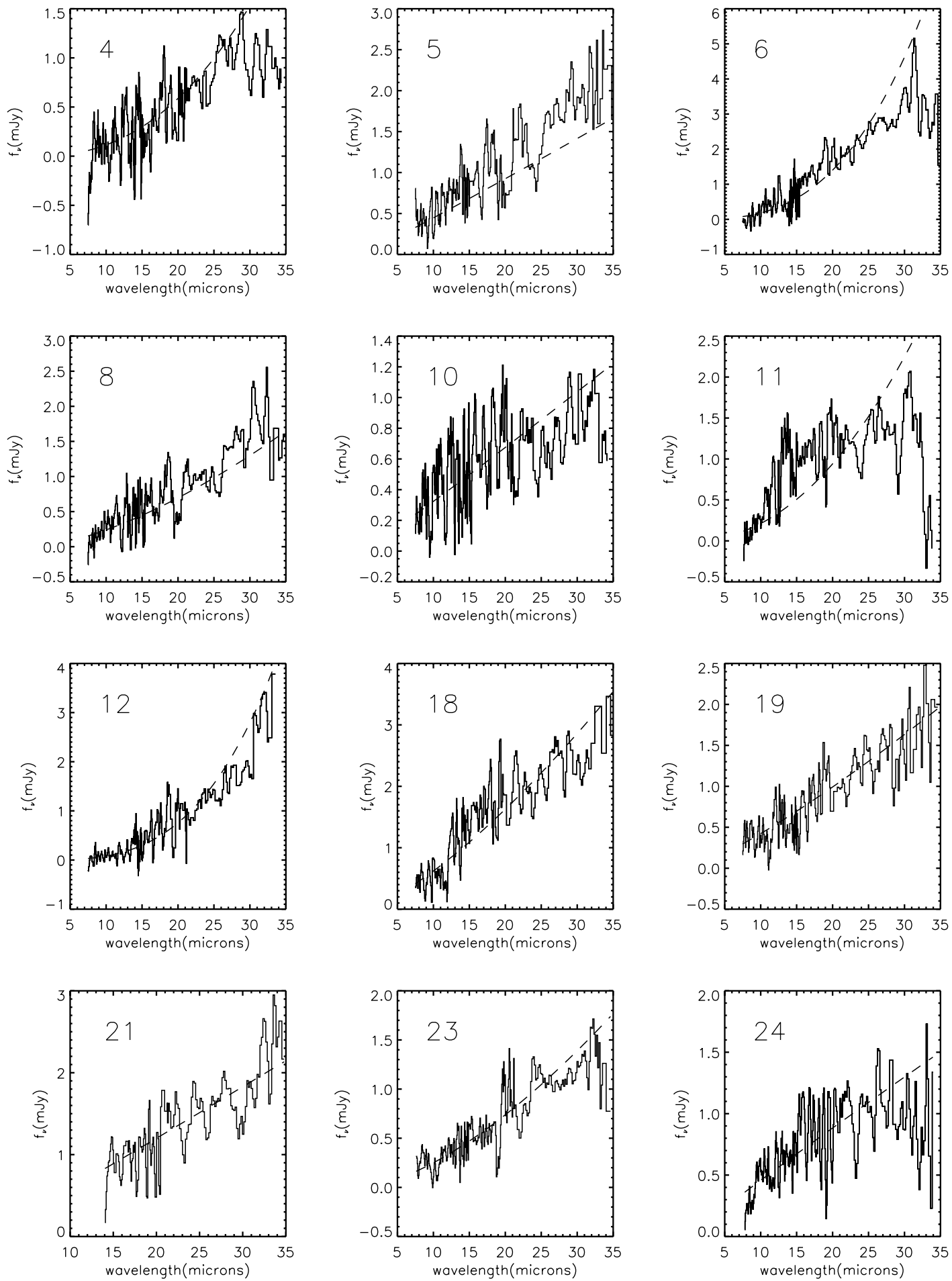

FIG. 2.-Observed IRS spectra of featureless sources in Table 1 (histogram). Spectra are smoothed to approximate resolution of individual IRS orders. Dashed curve: Power law that would connect MIPS $f_{\nu}(24 \mu \mathrm{m})$ with the $\operatorname{IRAC} f_{\nu}(8 \mu \mathrm{m})$.

at a range of air masses to correct for extinction. For objects 4 and 24 , the individual frames from June 19 were scaled to match the photometry from June 20, and all frames were then combined using variance weighting.

All seven objects were detected, with $18.6<K<20.9$ mag. The photometric uncertainties range from 0.1 to $0.3 \mathrm{mag}$ and are dominated by the source flux measurement, but include the zeropoint uncertainties from the standard stars. Sources 4, 9, 16, and 17 are clearly resolved.

\section{DISCUSSION}

\subsection{Characteristics of the Boötes Sample}

Understanding the full extragalactic population of mid-infrared sources is crucial to determining the evolution of dusty, obscured sources in the universe and to determining how those sources compare in number and evolution to optically visible sources. Already there are indications from IRS results for the Boötes sources that the high-redshift population of luminous, dusty 
TABLE 1

Observations and Properties of Sources

\begin{tabular}{|c|c|c|c|c|c|c|c|}
\hline Source & Name $^{\mathrm{a}}$ & $\begin{array}{c}f_{\nu}(24 \mu \mathrm{m})^{\mathrm{b}} \\
(\mathrm{mJy})\end{array}$ & $\begin{array}{c}\text { Time }(\mathrm{LL}, \mathrm{SL})^{\mathrm{c}} \\
(\mathrm{s})\end{array}$ & $\mathrm{IR} / \mathrm{opt}^{\mathrm{d}}$ & $\alpha^{\mathrm{e}}$ & $z^{\mathrm{f}}$ & $\mathrm{S} / \mathrm{N}^{\mathrm{g}}$ \\
\hline 1 & SST24 J142936.71+323857.1 & 1.27 & 1200,240 & 140 & $2.5,2.2,1.7$ & $2.4, \ldots$ & 3.6 \\
\hline 2 & SST24 J143102.26+325152.3 & 1.34 & 1200,240 & $>180$ & $0.9,2.5,1.4$ & $2.0,[1.2]$ & 5.1 \\
\hline 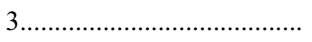 & SST24 J143026.05+331516.4 & 1.83 & 960,240 & 60 & $2.4,1.0,0.9$ & $1.9, \ldots$ & 4.5 \\
\hline 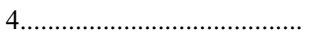 & SST24 J143318.59+332127.0 & 0.91 & 1680,240 & $>120$ & $3.0,2.4,1.0$ & $\ldots, \ldots$ & 4.1 \\
\hline 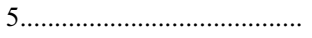 & SST24 J142644.34+333052.0 & 1.12 & 1440,240 & 50 & $2.2,1.1,1.3$ & $\ldots, \ldots$ & 4.5 \\
\hline 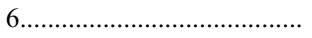 & SST24 J143308.62+333401.7 & 2.40 & 720,240 & 270 & $2.7,2.9,1.8$ & $\ldots, \ldots$ & 6.8 \\
\hline 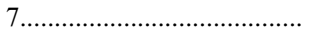 & SST24 J143053.14+334332.3 & 0.87 & 1680,240 & $>120$ & $1.9,1.6,-0.6$ & $\ldots, \ldots$ & 1.8 \\
\hline 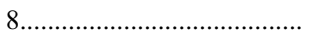 & SST24 J142920.47+334400.7 & 0.92 & 1680,240 & $>120$ & $3.3,1.5,1.7$ & $\ldots, \ldots$ & 4.1 \\
\hline 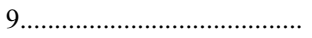 & SST24 J143424.50+334543.3 & 0.88 & 1680,240 & 50 & $2.4,1.9,1.7$ & $\ldots, \ldots$ & 2.8 \\
\hline 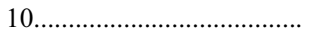 & SST24 J142827.95+334550.3 & 0.82 & 1920,240 & 100 & $2.0,1.1,0.9$ & $\ldots, \ldots$ & 2.9 \\
\hline 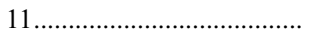 & SST24 J143253.39+334844.3 & 1.40 & 1200,240 & $>190$ & $3.8,2.1,0.4$ & $\ldots, \ldots$ & 4.0 \\
\hline 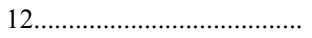 & SST24 J143004.77+340929.9 & 1.36 & 720,240 & 40 & $-0.9,3.2,2.9$ & $\ldots,[0.8]$ & 4.1 \\
\hline 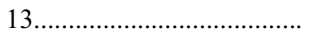 & SST24 J143807.96+341612.5 & 2.19 & 720,180 & $>300$ & $2.6,2.3,1.8$ & $2.2, \ldots$ & 4.7 \\
\hline 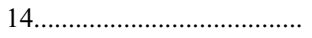 & SST24 J142745.88+342209.0 & 1.52 & 1680,240 & 110 & $3.0,1.8,1.8$ & $1.4, \ldots$ & 5.8 \\
\hline 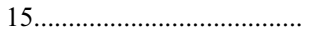 & SST24 J142842.96+342409.9 & 3.09 & 480,120 & 80 & $2.1,1.2,1.0$ & $1.1, \ldots$ & 7.7 \\
\hline 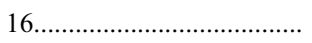 & SST24 J143429.56+343633.1 & 2.29 & 720,240 & 110 & $3.1,2.8,2.0$ & $2.0, \ldots$ & 6.5 \\
\hline 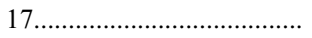 & SST24 J142940.85+344048.7 & 1.02 & 1440,240 & 80 & $2.7,2.2,1.3$ & $2.0, \ldots$ & 5.0 \\
\hline 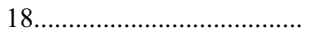 & SST24 J142748.47+344851.3 & 2.10 & 720,180 & 50 & $4.2,1.4,1.5$ & $\ldots, \ldots$ & 5.8 \\
\hline 19 & SST24 J143213.40+350802.1 & 1.24 & 1200,240 & $>170$ & $2.1,1.2,1.2$ & $\ldots, \ldots$ & 1.8 \\
\hline 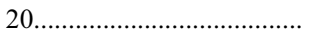 & SST24 J142611.38+351218.0 & 1.55 & 1200,240 & 50 & $2.5,1.4,0.7$ & $1.6, \ldots$ & 3.1 \\
\hline 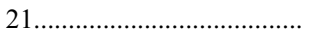 & SST24 J142759.92+351243.5 & 1.45 & 720,0 & $>200$ & $2.4,1.0,1.1$ & $\ldots, \ldots$ & 4.8 \\
\hline 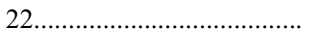 & SST24 J143546.13+352447.2 & 0.99 & 1440,240 & $>130$ & $2.1,0.8,1.0$ & $\ldots, \ldots$ & 2.8 \\
\hline 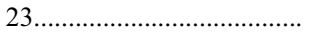 & SST24 J142850.94+353146.6 & 0.98 & 1440,240 & $>130$ & $3.2,1.6,1.4$ & $\ldots, \ldots$ & 4.5 \\
\hline 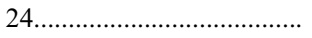 & SST24 J142939.18+353558.4 & 1.05 & 1440,240 & $>140$ & $2.7,0.9,-0.1$ & $\ldots, \ldots$ & 3.9 \\
\hline
\end{tabular}

${ }^{a}$ SST24 source name derives from discovery with the MIPS $24 \mu \mathrm{m}$ images; coordinates listed are J2000.0 $24 \mu \mathrm{m}$ positions with typical $3 \sigma$ uncertainty of \pm 1 1"2.

b Values of $f_{\nu}(24 \mu \mathrm{m})$ are for an unresolved point source, measured from MIPS images.

${ }^{c}$ First number is total integration time for each order of the Long-Low spectrum; second number is total integration time in Short-Low order 1.

${ }^{\mathrm{d}} \mathrm{IR} / \mathrm{opt}=\nu f_{\nu}(24 \mu \mathrm{m}) / \nu f_{\nu}(I)$. For Vega magnitudes, zero magnitude in $I$ band corresponds to $2450 \mathrm{Jy}$. Limits are based on assumed $I>25$. Values of $I$ are listed in Table 2 .

e $\alpha$ is the power-law index for different portions of the continuum of form $f_{\nu} \propto \nu^{-\alpha}$ : the first number is the power law index that would connect the IRAC $f_{\nu}(8 \mu \mathrm{m})$ with the IRAC $f_{\nu}(3.6 \mu \mathrm{m})$; the second number is the power law index that would connect the MIPS $f_{\nu}(24 \mu \mathrm{m})$ with the $\operatorname{IRAC} f_{\nu}(8 \mu \mathrm{m})$; and the third number is the $\alpha(14-33)$ for the continuum as determined from the best fit to the IRS spectra from $14 \mu \mathrm{m}$ to $33 \mu \mathrm{m}$.

${ }_{\mathrm{f}}^{\mathrm{f}}$ Redshifts are possible redshifts derived from fits of a weak silicate feature in the IRS spectra. Redshifts in brackets are estimated from a photometric $z$ based on inflection among the IRAC fluxes that can be fit with a template SED containing the $1.6 \mu \mathrm{m}$ peak in the spectrum of an evolved stellar component, shown in Fig. 6.

${ }^{\mathrm{g}}$ Signal-to-noise ratio as measured in LL1 from the best-fit continuum longward of $20 \mu \mathrm{m}$ by S/N $=f_{\nu}(27 \mu \mathrm{m}) / \mathrm{rms}$, for rms the $1 \sigma$ noise about the best-fit continuum.

galaxies that are optically obscured exceeds the population previously known from optical surveys. H05 argue that the obscured sources with IRS redshifts determined from silicate absorption derive their mid-infrared luminosity primarily from an active galactic nucleus (AGN) based on the high luminosities required, the similarity to infrared spectra of known local AGNs, and the occasional presence of strong radio sources. These IRS observations indicate that for redshifts $z \gtrsim 2$, the obscured quasars already discovered are a comparable population to unobscured quasars. This conclusion derives from comparison with quasars discovered in the same Boötes survey at similar $24 \mu \mathrm{m}$ fluxes but which are optically bright.

Using optically determined spectroscopic redshifts, Brown et al. (2005) derive the redshift distribution and luminosity function of optically classifiable quasars discovered in the MIPS $24 \mu \mathrm{m}$ Boötes survey. This result includes $87 \%$ of the Boötes sources having $f_{\nu}(24 \mu \mathrm{m})>1 \mathrm{mJy}$ and $R<21.7$ (for type 1 quasars, the I magnitude limit would be similar). This comprehensive sample has, therefore, a $24 \mu \mathrm{m}$ selection criterion very similar to that of our sample but much brighter optical magnitudes; the IR/opt for the Brown et al. sample is what is expected for unobscured, type 1 quasars. Brown et al. conclude quantitatively that the shape of the rest-frame $8 \mu \mathrm{m}$ infrared luminosity function is the same as derived from optical surveys and that the redshift peak of quasar space density is at the same redshift. In sum, selection of unobscured quasars based on $24 \mu \mathrm{m}$ fluxes gives the same result for comparable optical magnitude limits as a sample based on optical criteria alone. There are 35 quasars in the full Boötes field with $1.9<z<2.7$ in their nearly complete sample.

These numbers can be compared directly with the characteristics of our optically obscured sample within the same redshift interval by considering only those sources with $1.9<z<2.7$ selected to the same infrared limit, having $f_{\nu}(24 \mu \mathrm{m})>1 \mathrm{mJy}$, but with $I>24$ mag. There are 14 sources with IRS spectra observed in Boötes that meet these redshift and flux criteria. There are a total of 65 objects in the full Boötes field satisfying both of these optical and infrared flux criteria, and 35 of these 65 were observed with the IRS. Approximately, therefore, the 14 sources with redshifts $1.9<z<2.7, f_{\nu}(24 \mu \mathrm{m})>1 \mathrm{mJy}$, and $I>24$ mag derive from $35 / 65$, or $\sim 54 \%$, of a complete sample defined by $f_{\nu}(24 \mu \mathrm{m})>1 \mathrm{mJy}$ and $I>24 \mathrm{mag}$. Correcting for this factor of $\sim 2$ incompleteness, there should be $\sim 27$ optically faint sources $(I>24 \mathrm{mag})$ having $1.9<z<2.7$ in the full Boötes field. This number is comparable to the 35 unobscured, type 1 quasars within this redshift range that are known in the field from Brown et al. (2005). This comparison gives an empirical indication that optically obscured sources already identified at these high redshifts are similar in number to the classical, optically discoverable type 1 AGNs. This is only a lower limit to the actual number of obscured sources, because the 24 objects in the Boötes sample without redshifts are not included, and partially obscured sources having $f_{\nu}(24 \mu \mathrm{m})>1 \mathrm{mJy}$ in the intermediate 
TABLE 2

Near-Infrared Flux Densities and Optical Magnitudes for Sources

\begin{tabular}{|c|c|c|c|c|c|c|c|c|c|}
\hline Source & SST24 Source Name & $\begin{array}{c}f_{\nu}(3.6 \mu \mathrm{m})^{\mathrm{a}} \\
(\mu \mathrm{Jy})\end{array}$ & $\begin{array}{c}f_{\nu}(4.5 \mu \mathrm{m})^{\mathrm{a}} \\
(\mu \mathrm{Jy})\end{array}$ & $\begin{array}{c}f_{\nu}(5.8 \mu \mathrm{m})^{\mathrm{a}} \\
(\mu \mathrm{Jy})\end{array}$ & $\begin{array}{c}f_{\nu}(8 \mu \mathrm{m})^{\mathrm{a}} \\
(\mu \mathrm{Jy})\end{array}$ & $\begin{array}{c}B_{W}^{\mathrm{b}} \\
(\mathrm{mag})\end{array}$ & $\begin{array}{c}R^{\mathrm{b}} \\
(\mathrm{mag})\end{array}$ & $\begin{array}{c}I^{\mathrm{b}} \\
(\mathrm{mag})\end{array}$ & $\begin{array}{c}K^{\mathrm{c}} \\
(\mathrm{mag})\end{array}$ \\
\hline 1 & $\mathrm{~J} 142936.71+323857.1$ & 15.8 & 27.6 & 33.4 & 114 & 26.5 & 25.2 & 24.8 & \\
\hline 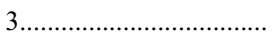 & $\mathrm{J} 143026.05+331516.4$ & 91 & 162 & 358 & 622 & 24.7: & 24.3: & 23.4: & $\ldots$ \\
\hline 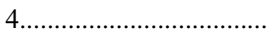 & $\mathrm{J} 143318.59+332127.0$ & 6.0 & 7.3 & 33.1 & 68.4 & $\ldots$ & $\ldots$ & $\ldots$ & $\ldots$ \\
\hline 5 & $\mathrm{~J} 142644.34+333052.0$ & 60.4 & 84 & 161 & 353 & 26.4: & 24.5: & 23.8: & 18.6 \\
\hline 8 & $\mathrm{~J} 142920.47+334400.7$ & 12.2 & 22.9 & 8.3 & 170 & $\ldots$ & $\ldots$ & $\ldots$ & 20.9 \\
\hline 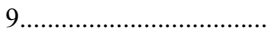 & $\mathrm{J} 143424.50+334543.3$ & 15.7 & 21.1 & 79 & 107 & $\ldots$ & $\ldots$ & 24.0: & 19.8 \\
\hline 10 & $\mathrm{~J} 142827.95+334550.3$ & 54.0 & 83 & 139 & 257 & 25.4 & 25.5 & 24.9 & $\ldots$ \\
\hline 11 & $\mathrm{~J} 143253.39+334844.3$ & 6.5 & 6.6 & 21.5 & 133 & $\ldots$ & $\ldots$ & $\ldots$ & $\ldots$ \\
\hline 12 & $\mathrm{~J} 143004.77+340929.9$ & 85 & 50.6 & 38.7 & 39.8 & 26.0 & 23.9 & 23.2 & $\ldots$ \\
\hline $13 \ldots \ldots$ & $\mathrm{J} 143807.96+341612.5$ & 21.0 & 28.1 & 72 & 168 & $\ldots$ & 25.5 & $\ldots$ & $\ldots$ \\
\hline 14 & $\mathrm{~J} 142745.88+342209.0$ & 18.6 & 39.9 & 98 & 212 & 24.8 & 24.2 & 24.3 & $\ldots$ \\
\hline 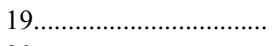 & $\mathrm{J} 143213.40+350802.1$ & 58.7 & 95 & 188 & 323 & 26.4 & $\ldots$ & $\ldots$ & $\ldots$ \\
\hline 20 & $\mathrm{~J} 142611.38+351218.0$ & 48.0 & 76 & 172 & 350 & 25.1 & 23.8 & 23.3 & $\ldots$ \\
\hline 21 & $\mathrm{~J} 142759.92+351243.5$ & 67 & 111 & 239 & 463 & 25.1 & 24.4 & $\ldots$ & $\ldots$ \\
\hline 22 & $\mathrm{~J} 143546.13+352447.2$ & 71 & 111 & 188 & 395 & 25.4 & 26.0 & $\ldots$ & $\ldots$ \\
\hline $23 \ldots \ldots$ & $\mathrm{J} 142850.94+353146.6$ & 13.9 & 29.4 & 87 & 179 & $\ldots$ & $\ldots$ & $\ldots$ & $\ldots$ \\
\hline 24 & J142939.18+353558.4 & 43 & 85 & 168 & 371 & $\ldots$ & $\ldots$ & $\ldots$ & 20.1 \\
\hline
\end{tabular}

\footnotetext{
${ }^{a}$ Fluxes from IRAC measures, assuming a point source.

${ }^{\mathrm{b}}$ Sources with an optical counterpart appear in NDWFS catalogs with prefix NDWFS and the optical source position; NDWFS Data Release 3 (DR3) is available at http://www.archive.noao.edu/ndwfs/. Magnitudes are Vega magnitudes for an unresolved source, measured from images in NDWFS DR3 by smoothing all images to a PSF of FWHM 1".35 and applying photometry at the $24 \mu \mathrm{m}$ source position with an aperture of 4 " diameter, except for objects with magnitudes followed by colon, which use $3^{\prime \prime}$ apertures because of proximity to a brighter source. The aperture photometry has been corrected to the total magnitude for an unresolved source by a correction of $0.1 \mathrm{mag}$ brighter for the $4^{\prime \prime}$ aperture and 0.3 mag brighter for the $3^{\prime \prime}$ aperture.

${ }^{c} K$ magnitudes are total magnitudes.
}

magnitude range $22 \lesssim I \lesssim 24$ have not been counted in this tally of obscured AGNs. Our result that the number density of obscured quasars exceeds that of unobscured quasars at high redshifts is also consistent with estimates of obscured AGNs derived using Spitzer infrared colors compared with optical or radio classifications of AGNs (Alonso-Herrero et al. 2006; Martinez-Sanigre et al. 2005).

To refine our measures of the obscured population, it is necessary to understand whether the 24 sources without IRS redshifts are also high-redshift, obscured sources. There are two alternative explanations for why strong spectral features are not visible in these 24 sources. The first is that the redshifts are so high that features are shifted out of the range of the IRS. The highest redshift for which the silicate absorption feature is measured in the initial results of $\mathrm{H} 05$ is 2.73 . If sources have redshifts above 2.8 , the silicate absorption feature would be centered at observed wavelength of $37 \mu \mathrm{m}$, so even the beginning of this feature could not be confidently identified in IRS spectra of poor S/N. There is one observed example already of a source with known redshift higher than this limit that shows a featureless spectrum within the IRS wavelengths. This is the gravitationally lensed quasar APM $08279+5255$, having $z=3.91$, which shows a featureless power law over the full IRS range corresponding to rest wavelengths of 1.7-7 $\mu \mathrm{m}$ (Soifer et al. 2004), with a power-law slope of 1.5. By comparison to this source, we would not expect to see features in sources with $z \gtrsim 2.8$.

The alternative explanation is that sources have redshifts that place the $9.7 \mu \mathrm{m}$ silicate absorption feature within our spectral range, but that this feature is too weak to be detected. The $\mathrm{S} / \mathrm{N}$ required to see such features depends on the strength of the feature, so we can never rule out the presence of sufficiently weak features in our spectra. However, we had already determined that features at the level of the weakest silicate absorption feature in any of the AGN templates used for the redshift determinations in H05 are not present. Templates used were Arp 220, IRAS F001837111, and Mrk 231; the weakest $9.7 \mu \mathrm{m}$ absorption feature within these templates is Mrk 231. For Mrk 231, the absorption depth is $50 \%$ (the IRS spectrum is in Weedman et al. 2005); i.e., the absorption has removed $50 \%$ of the unabsorbed continuum that would otherwise be present at the wavelength of maximum absorption. The absorption depth, defined as ABS $=[1-f(9.7) / f(\mathrm{CONT})]$, is estimated by comparing the observed flux density at $9.7 \mu \mathrm{m}$, $f(9.7)$, with the continuum flux density, $f(\mathrm{CONT})$, predicted for an unabsorbed continuum as determined from a linear fit to the continuum level on either side of the silicate feature. The fraction of the continuum that is absorbed relates to the optical depth as $\mathrm{ABS}=1-e^{-\tau}$, where $\tau$ is the maximum optical depth in the $9.7 \mu \mathrm{m}$ silicate absorption feature. The six sources with the weakest features in $\mathrm{H} 05$ are fit with the Mrk 231 template, which has $\mathrm{ABS}=0.5$; the remaining 11 sources have $\mathrm{ABS}>0.5$.

The detectability of silicate absorption depends on the value of ABS. Of the 58 sources in Boötes observed in our faint-source sample, the 24 sources discussed in the present paper, or $41 \%$, are those with $\mathrm{ABS}<0.5$; they did not have sufficient absorption to be fit with a template that would yield a confident redshift. The sample of Boötes sources was chosen because of the extreme IR/opt, and we do not have a control sample of objects with known redshifts selected in the same way to determine what 
fraction of such sources would be expected to have deep silicate absorption. The best set of such comparison sources currently available is a large sample of ultraluminous infrared galaxies (ULIRGS) observed by the IRS (Spoon et al. 2006). We have examined IRS low-resolution spectra of 80 ULIRGS in this sample to estimate the depth of the $9.7 \mu \mathrm{m}$ silicate absorption feature (or height of any broad emission features, if present). Of the 80 sources, 60 (75\%) show the silicate absorption feature with absorption depth ABS $>0.5$ (L. Hao et al. 2006, in preparation). This result emphasizes the prevalance of deep absorption among ULIRGS and implies that absorption should be characteristic of dusty sources. If the Boötes sources are all at redshifts such that the $9.7 \mu \mathrm{m}$ absorption feature is accessible to the IRS, we expect them to show absorption as frequently as do ULIRGS, given the Boötes selection designed to favor obscured sources. That only $60 \%$ of the Boötes sources show ABS $>0.5$ compared to $75 \%$ of ULIRGS is not a statistically significant difference given the sizes of the two samples, so this comparison is consistent with (although does not prove) the interpretation that all of the Boötes sources are sources similar to absorbed ULIRGS.

Because Mrk 231 is being used as a comparison template for these sources, it is useful to ask how it would appear if at $z=2$. The redshift is 0.0422 , and the observed $f_{\nu}(8.4 \mu \mathrm{m})=1080 \mathrm{mJy}$ (Rieke 1976), so the observed $f_{\nu}(24 \mu \mathrm{m})$ if at $z=2$ would be $0.4 \mathrm{mJy}$. The IR/opt is more uncertain because a total magnitude is not available at rest-frame $0.27 \mu \mathrm{m}$ for comparison to the observed $I$ band at $z=2$. The observed flux density at rest-frame $0.34 \mu \mathrm{m}$ is $4 \mathrm{mJy}$ (Surace \& Sanders 2000); the rest-frame flux density at $0.27 \mu \mathrm{m}$ for the nucleus alone, without correction for a total galaxy magnitude, is $0.5 \mathrm{mJy}$ (Smith et al. 1995). These results bracket the true total magnitude at $0.27 \mu \mathrm{m}$ and so yield that Mrk 231 would have $9<\mathrm{IR} /$ opt $<72$ if it were at $z=2$. For scaling, a source of $I=24$ and $f_{\nu}(24 \mu \mathrm{m})=1 \mathrm{mJy}$ has an IR/opt of 60 . The sources in Table 1 have an IR/opt $\geq 40$, and $75 \%$ of these sources have an IR/opt greater than the maximum value that Mrk 231 could have. Overall, therefore, Mrk 231, if at $z=2$, would be too faint for our flux selection criterion for Boötes but might meet the IR/opt criterion. It is sufficiently similar, however, to indicate that it is a valid comparison source.

\subsection{Comparison of Boötes Sources with and without Strong Spectral Features}

To consider whether the Boötes sources with weak spectral features are systematically different from the heavily absorbed sources with previously measured redshifts, we compare the samples and look more closely at detailed spectral characteristics including overall SEDs. The first test is to consider whether there are any obvious systematic differences between the Boötes sources with redshifts determined from deep absorption (ABS > 0.5) and those sources in the present paper without strong spectral features. Figure 3 shows the distributions of IR/opt and $f_{\nu}(24 \mu \mathrm{m})$ for the no- $z$ sample in the present paper compared to the objects in $\mathrm{H} 05$ with redshifts. The median $f_{\nu}(24 \mu \mathrm{m})$ is $1.1 \mathrm{mJy}$ for those with redshifts and $1.3 \mathrm{mJy}$ for those without. These flux limits are similar, implying that the ability to determine a redshift does not depend on flux. (In fact, observations were designed with the goal of having similar $\mathrm{S} / \mathrm{N}$ at all fluxes, so that shorter integrations were used on brighter sources.) Counting the limits, sources with redshifts have median IR/opt of 145 and those without have 115. This slight difference in the distributions for IR/opt implies that the sources without redshift may be somewhat less obscured optically compared to those with deep silicate features that allowed redshift determinations. However, the difference would correspond to an optical magnitude difference of only

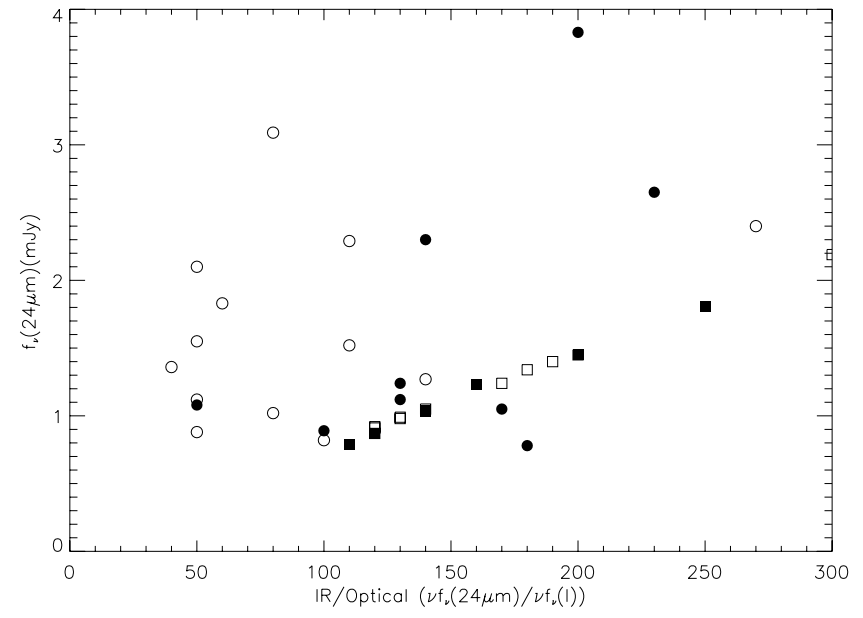

FIG. 3.-Values of $f_{\nu}(24 \mu \mathrm{m})$ and IR/opt $=\nu f_{\nu}(24 \mu \mathrm{m}) / \nu f_{\nu}(I)$ for sources in Table 1; measured IR/opt is shown as open circles, and lower limits on IR/opt as open squares. For comparison, $24 \mu \mathrm{m}$ flux densities and IR/opt are also shown for sources in H05, which have redshifts; measured IR/opt is shown as filled circles and lower limits on IR/opt as filled squares.

$0.25 \mathrm{mag}$, which is not a large difference compared to magnitude uncertainties. In any case, the no-z sources are much more optically obscured than type $1 \mathrm{AGN}$, which is the category of AGN that does not typically show silicate absorption; the optically bright, type 1 quasars in the $1 \mathrm{mJy}$ sample in Brown et al. (2005) have $1 \lesssim I R / o p t \lesssim 10$. The no- $z$ sample remains puzzling, therefore. How can sources that are seemingly very obscured optically have no strong absorption feature in the infrared?

Another comparison of the samples with and without redshifts is through the near-infrared colors. Such colors have been used to preselect targets based on models of how colors derive for starbursts and AGNs at various redshifts (Yan et al. 2005) and in empirical comparisons of AGNs and starbursts as classified by other criteria (Brand et al. 2006). Figure 4 shows the distributions of colors among MIPS 24 and IRAC $8 \mu \mathrm{m}$ and $3.6 \mu \mathrm{m}$ fluxes. There is little difference between the Boötes samples with and without redshifts. The median $\log \left[f_{\nu}(24 \mu \mathrm{m}) / f_{\nu}(8 \mu \mathrm{m})\right]$ is 1.0 for sources with redshifts, and 0.85 for sources without, indicating similar mid-infrared continuum slopes for both samples, which implies similar distributions of dust temperatures among the samples.

While this result does not distinguish the two samples, the slopes for both samples are steep enough to expect that many sources should be starbursts based on the spectral slopes. Yan et al. (2005) considered a value of $\log \left[f_{\nu}(24 \mu \mathrm{m}) / f_{\nu}(8 \mu \mathrm{m})\right]=1.0$ as dividing sources with starburst spectra $(>1.0)$ from those with AGN spectra $(<1.0)$. By this criterion, about half of the objects in Figure 4 should be starbursts. As previously noted, however, only 1 of the 24 Boötes sources in Figures 1 and 2 possibly shows $\mathrm{PAH}$ features, and it is these features that are associated with starbursts (Genzel et al. 1998).

Brand et al. (2006) have recently demonstrated that AGNs identified because of X-ray luminosity have a different distribution of mid-infrared colors compared to other mid-infrared sources. They compare the IRAC and MIPS fluxes for all sources in the Boötes survey with the $\sim 5 \%$ of sources detected by a Chandra $X$-Ray Observatory survey within the Boötes field. Brand et al. show that the X-ray sources are distributed around a value of $\log \left[f_{\nu}(24 \mu \mathrm{m}) / f_{\nu}(8 \mu \mathrm{m})\right]=0.5$, with a $1 \sigma$ of 0.16 for the width of the distribution, whereas the remaining sources are distributed about a value of 1.0. With this criterion, only half of the Boötes sources in Figure 4 fall within the $3 \sigma$ distribution for an 


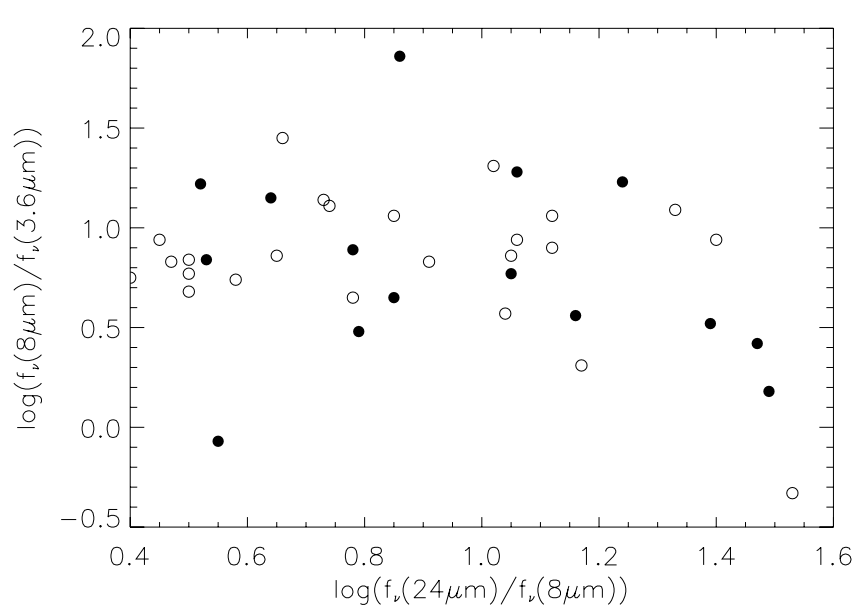

FIG. 4.-Distributions of $\log \left[f_{\nu}(8 \mu \mathrm{m}) / f_{\nu}(3.6 \mu \mathrm{m})\right]$ and $\log \left[f_{\nu}(24 \mu \mathrm{m}) /\right.$ $\left.f_{\nu}(8 \mu \mathrm{m})\right]$ for sources in Table 1 (open circles); shown for comparison are sources with redshifts in $\mathrm{H} 05$ (filled circles).

X-ray-luminous AGN. This again raises the question of why the Boötes sources with spectral slopes steeper than those of AGNs rarely show evidence in the IRS spectra of the PAH features from starbursts.

One explanation might be that these Boötes sources with steep slopes but no PAH features are AGNs, which have low X-ray luminosity and systematically cooler dust than the AGNs associated with detectable X-ray sources. An alternative explanation is that the sources with steep slopes but no PAH emission are not powered by AGNs, but are powered by a starburst even though there is no PAH emission. In this circumstance, the starburst would have to be so embedded in dust or of sufficiently low metallicity that the PAH emission is absorbed or not produced, as suggested by Hunt \& Maiolino (2005) to be characteristic of primordial starbursts and as observed for the blue compact galaxy SBS 0335-052 ( Thuan et al. 1999; Houck et al. 2004b). Regardless of the explanation, the empirical conclusion is that the $f_{\nu}(24 \mu \mathrm{m}) / f_{\nu}(8 \mu \mathrm{m})$ does not give a consistent classification compared to mid-infrared spectral features for determining whether sources have their mid-infrared luminosity derived from an AGN or from a starburst.

Using the photometry from the NDWFS together with the IRAC and MIPS fluxes, the overall SEDs of the sources can be examined. Most of the sources in the Boötes sample have SEDs that are monotonically decreasing from the MIPS $24 \mu \mathrm{m}$ flux density through all four IRAC bands and the optical bands. For such sources, these photometric continuum fluxes can be fit by a single power law or by a power law that becomes steeper at shorter wavelengths. For the entire Boötes sample of 58 sources, 41 sources $(71 \%)$ have power-law spectra and so can be interpreted as having all infrared fluxes dominated by a dust continuum. The remaining 17 sources show inflections among the IRAC points, indicating the possible presence of a stellar component. For the 34 sources with redshifts, 25 are power laws. For the 24 no-z sources in the present paper, 16 are power laws. In this respect, the two samples are also similar.

These comparisons of IR/opt, mid-infrared colors, and SEDs do not indicate any differences in characteristics between the Boötes sources with strong absorption features and those with weak features. Consequently, these comparisons do not indicate a conclusion regarding whether the no- $z$ sources are at $z \lesssim 2.8$ or $z \gtrsim 2.8$.

Even though some sources do not have sufficiently strong silicate absorption to be detected in our spectra, sources with weak silicate absorption could have sufficient optical absorption to produce large values of IR/opt. As previously emphasized, the sources in the Boötes samples were selected to be optically faint, $I \geq 24 \mathrm{mag}$, and to have extreme values of IR/opt. At redshift of 2 , the rest-frame ultraviolet at $0.27 \mu \mathrm{m}$ observed in the $I$ band is heavily extincted compared to the extinction required to produce the $9.7 \mu \mathrm{m}$ absorption feature. Using the grain model of $\mathrm{Li} \&$ Draine (2001), the ratio of extinctions is $A(0.27 \mu \mathrm{m}) / A(9.7 \mu \mathrm{m})=$ 24 , where $A$ is the extinction in magnitudes. A silicate absorption feature with $\mathrm{ABS}=0.10$, or optical depth of 0.1 in the $9.7 \mu \mathrm{m}$ feature, has $A(9.7 \mu \mathrm{m})=0.11 \mathrm{mag}$, so $A(0.27 \mu \mathrm{m})=2.7 \mathrm{mag}$. This means that an unobscured, type 1 quasar of $I=22 \mathrm{mag}$ would be extincted to $I>24 \mathrm{mag}$ if the ultraviolet continuum were absorbed by the same dust that produces a $9.7 \mu \mathrm{m}$ absorption feature of ABS $=0.10$.

A source with $\mathrm{ABS}=0.10$ would not have a sufficiently strong absorption feature to have been measured within the available Boötes spectra. That such a source could nevertheless have significant optical extinction is consistent with the explanation that all of the Boötes sources are obscured sources with large values of IR/opt arising from dust extinction, even though the $9.7 \mu \mathrm{m}$ feature was not detected in all of the IRS spectra. This interpretation would also explain why the IR/opt does not correlate with the detection of $9.7 \mu \mathrm{m}$ absorption; a source with negligible ABS can still have sufficient ultraviolet extinction to fall within a sample having $I>24 \mathrm{mag}$. This conclusion indicates that properties of the sources with weak silicate features are consistent with those of obscured AGNs, but it is not proof of that classification. It remains crucial to detect any weak absorption features within the spectra; this is discussed in $\S 3.3$.

\subsection{Possible New Spectroscopic Redshifts}

To examine the spectra more carefully in a search for the possible presence of weak spectral features, we fit the power law that would connect the IRAC $(8 \mu \mathrm{m})$ and MIPS $(24 \mu \mathrm{m})$ photometry, and display this continuum in Figures 1 and 2. By seeking departures from this power law, we can attempt to determine whether or not real spectral features are present. As previously emphasized, none of these sources are characterized by the readily visible, strong absorption features that allowed redshift measurements for other sources with similar S/ N in H05.

Sources are divided into Figure 1 and Figure 2 by placing in Figure 1 those that appear to have weak features that might be real. The 12 spectra in Figure 1 have been fit with varying combinations of a power law and a silicate absorption profile ( $\mathrm{Li} \&$ Draine 2001). Initially, the best-fitting power law to the entire IRS spectrum is determined by adopting the least-squares fit with the minimum residuals. Then, the best-fitting combination of a power law of varying slope with a silicate profile of varying depth is fit by stepping through all redshifts $1<z<3$ in increments of 0.1 . Results are compared for goodness of fit between the power-law fits and the power-law plus absorption fits. For 10 of the 12 sources in Figure 1, the fit containing absorption is quantitatively better. An example is shown in Figure 5, for source 17. The absorption fit shown has a redshift of 2.0 and $\mathrm{ABS}=0.25$.

The sources in Figure 1 are now discussed individually, including comments on this absorption fit, and redshifts are listed in Table 1 when the fit using a weak silicate feature is statistically better than a power-law fit.

Source 1.- The spectral turnover starting at $\sim 25 \mu \mathrm{m}$ could be weak silicate absorption following the spectral peak at rest frame $8 \mu \mathrm{m}$ that is characteristic of absorbed sources; as seen in Mrk 231 and other absorbed ULIRGS, this spectral peak is not emission 

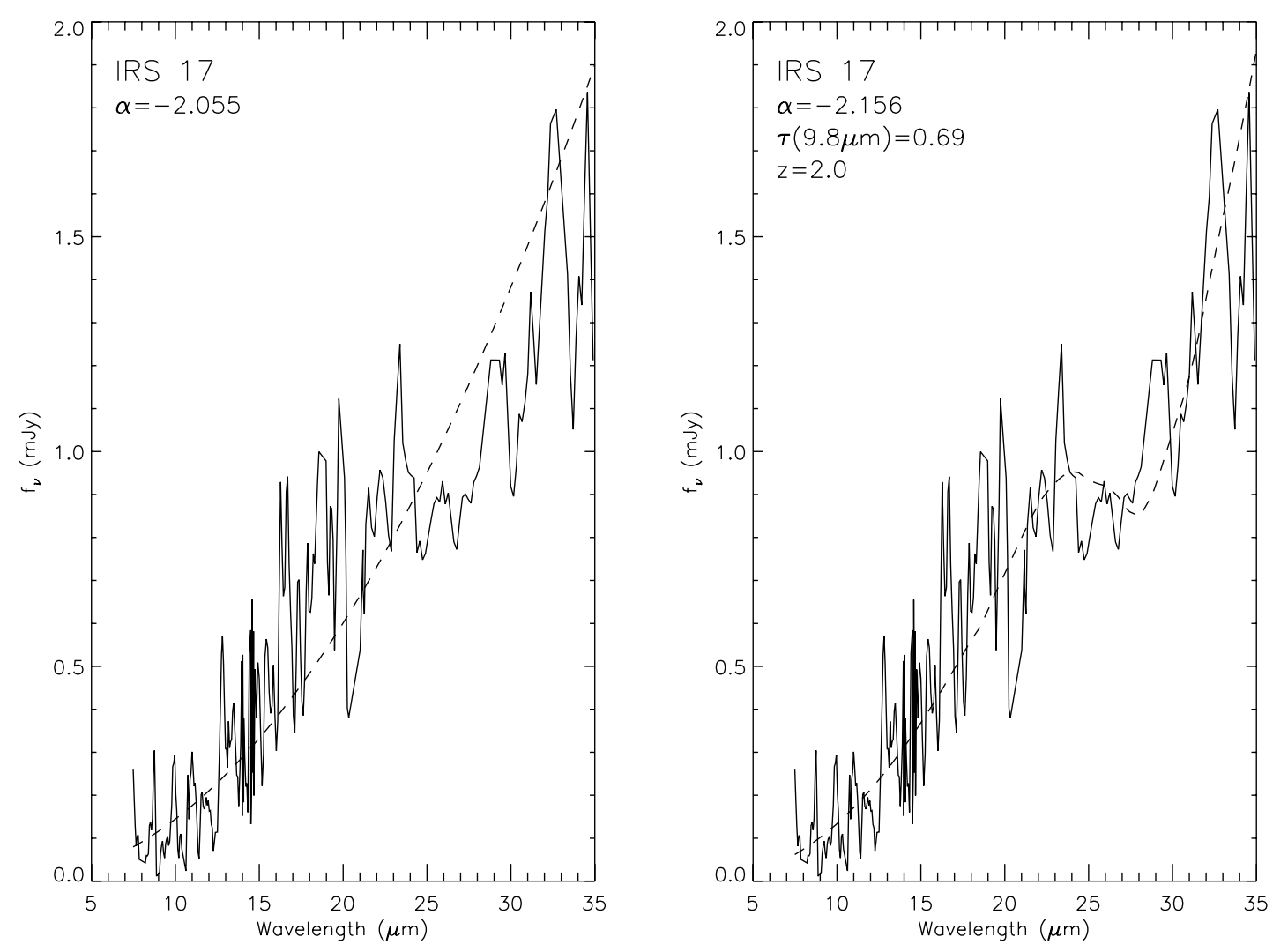

FIG. 5.-Left: IRS spectrum of source 17 (solid curve) with power-law fit (dashed curve). Right: IRS spectrum of source 17 (solid curve) with power-law fit plus a silicate absorption feature and redshift 2.0 (dashed curve).

but arises because of absorption at longer and shorter wavelengths. Fitting the spectrum with a power law and the silicate feature yields an optimum fit with $\mathrm{ABS}=0.28$ at $z=2.4$.

Source 2.-The spectral turnover starting at $\sim 23 \mu \mathrm{m}$ could be absorption, which can be fit by a silicate feature of $\mathrm{ABS}=0.45$ at $z=2.0$, but the photometric $z$ (discussed below) is 1.2. The photometric $z$ would be consistent with the broad peak centered at $\sim 20 \mu \mathrm{m}$ being silicate emission at rest-frame $10 \mu \mathrm{m}$ instead of the $8 \mu \mathrm{m}$ peak, but this would raise the puzzle of why an optically obscured source shows the silicate emission primarily found in unobscured, type 1 AGNs.

Source 3.- The possible absorption beyond $\sim 22 \mu \mathrm{m}$ can be fit by a silicate feature of ABS $=0.34$ at $z=1.9$.

Source 7.- The possible absorption beyond $\sim 21 \mu \mathrm{m}$ can be fit by a silicate feature of $\mathrm{ABS}=0.15$ at $z=1.8$, but this fit is not statistically better than a power-law fit without absorption, so a possible redshift is not listed in Table 1.

Source 9.-The peak at $\sim 20 \mu \mathrm{m}$ could be the $8 \mu \mathrm{m}$ peak, enhanced by noise, followed by very noisy absorption. The $\sim 20 \mu \mathrm{m}$ peak could also be $7.7 \mu \mathrm{m}$ PAH emission at $z \sim 1.6$, but we should then see the $11.2 \mu \mathrm{m}$ PAH feature at $29 \mu \mathrm{m}$, which is not present. A formal fit restricted to silicate absorption gives $z \sim 1.0$. This spectrum is so noisy that no weight is given to this result, and the redshift of this object is considered to be indeterminate.

Source 13.-There is a reasonably convincing $8 \mu \mathrm{m}$ peak at $\sim 24 \mu \mathrm{m}$ followed by absorption, and this can be fit by a silicate feature of $\mathrm{ABS}=0.30$ at $z=2.2$.

Source 14.- The peak at $\sim 20 \mu \mathrm{m}$ could be the $8 \mu \mathrm{m}$ peak, and the absorption can be fit by a silicate feature of $\mathrm{ABS}=0.39$ at $z=1.4$.

Source 16.- The fit with the standard silicate absorption profile fits the broader absorption beginning at $24 \mu \mathrm{m}$, for a silicate depth of $\mathrm{ABS}=0.29$ and $z=2.0$. In this case, the narrower, deeper absorption at $21 \mu \mathrm{m}$ is attributed to a noise feature.

Source 17.- This example of fitting the silicate feature is illustrated in Figure 5. The possible absorption beginning at $\sim 23 \mu \mathrm{m}$ is fit by silicate absorption of $\mathrm{ABS}=0.25$ and $z=2.0$.

Source 20 . - The peak at $\sim 21 \mu \mathrm{m}$ is reasonably convincing as being the $8 \mu \mathrm{m}$ peak followed by absorption, leading to a silicate fit of $\mathrm{ABS}=0.27$ and $z=1$.6. If this peak is instead the $7.7 \mu \mathrm{m}$ PAH emission, for $z \sim 1.7$, the $11.2 \mu \mathrm{m}$ PAH emission would correspond to the weaker feature at $29 \mu \mathrm{m}$, but the feature at $31 \mu \mathrm{m}$ would then have to be noise.

Source 22.- The $\mathrm{S} / \mathrm{N}$ is so poor for this source that the possible absorption beginning at the peak at $\sim 19 \mu \mathrm{m}$ cannot be fit by the power law-plus-silicate combination, so no redshift estimate is derived.

Suggestions for new redshift assignments are listed in Table 1. If we accept the suggested redshifts based on weak silicate absorption for the 9 sources in which this is identified (sources $1,2,3,13,14,15,16,17$, and 20), that brings to 43 the total number of spectroscopic redshift determinations for the 58 sources observed in Boötes.

There are a few objects in Figure 2 that might be questioned as to why we do not assign suggested features. For source 6 , little weight was given to the possible spectral turnover at $\sim 26 \mu \mathrm{m}$ because the presence of absorption would depend on assuming that the sharp peak at $\sim 32 \mu \mathrm{m}$ represents continuum, but this peak is where noise features are often found. A similar objection applies to the apparent absorption at $\sim 30 \mu \mathrm{m}$ in source 12 . For source 10 , the $\mathrm{S} / \mathrm{N}$ is so poor that the possible absorption at $\sim 26 \mu \mathrm{m}$ cannot be meaningfully fit. For source 11, the overall 

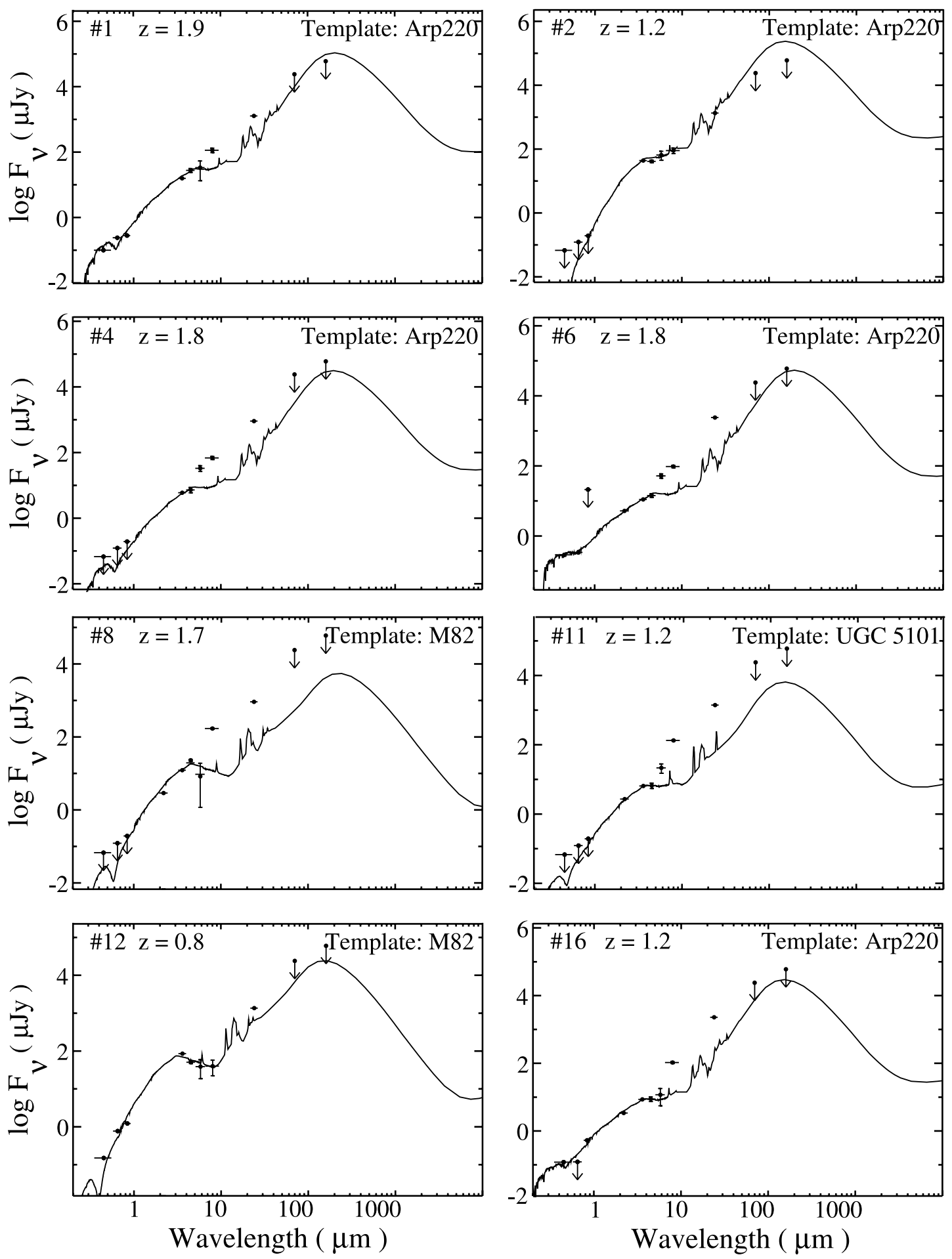

FIG. 6.-Spectral energy distributions for sources in Table 2 that show IRAC fluxes consistent with a photospheric bump that allows a photometric redshift estimate. Filled circles: Observed photometric data points. Solid curve: Template fit that leads to value of photometric redshift shown in panel.

flatness of the spectrum resembles a PAH emission spectrum, but no consistent redshift arises from the apparent emission features, so some or all of these features must be noise features, and no redshift is assigned.

\subsection{Spectral Energy Distributions and Photometric Redshifts}

Even when features allowing a spectroscopic redshift determination are not present, it is sometimes possible to estimate a photometric redshift for faint infrared sources. The estimate of a photometric redshift depends on identifying the redshifted peak in flux density at rest wavelength $\sim 1.6 \mu \mathrm{m}$ caused by the continuum maximum in giant or supergiant stars (Simpson $\&$ Eisenhardt 1999). For the 24 no- $z$ sources in the present paper, 16 are power laws consistent with an AGN SED (Polletta et al. 2006), so no photospheric peak is present, and no photometric redshift estimate is possible. There are eight sources having IRAC inflections that deviate from a power law that can be fit with templates containing a stellar photospheric component. SEDs of these eight sources are shown in Figure 6, with template fits that yield a photometric redshift. The template SEDs that are 
shown are intended only to be representative and to illustrate photometric estimates of redshift based on the $1.6 \mu \mathrm{m}$ peak. We did not attempt to determine which models optimize the SED fit or provide a quantitative separation of stellar luminosity and dust luminosity.

For most of the sources in Figure 6, the photospheric component is not conspicuous, presumably because a power-law component of dust continuum contributes to the flux at the wavelengths of the IRAC points and dilutes the photospheric component. This is seen in Figure 6 for sources 1, 4, 6, 8, 11, and 16 , for which the observed photometry at longer wavelengths exceeds the template. Only for sources 2 and 12 do the observed fluxes at all wavelengths fit a full template including the photospheric component, so a photometric redshift estimate is more reliable for these two sources and is given in Table 1. However, source 2 also has a redshift from a weak silicate feature (discussed above), which does not agree with the photometric redshift. For the remaining six sources with evidence of a photospheric component, the redshift of the $1.6 \mu \mathrm{m}$ peak is much more uncertain, and photometric redshifts are not adopted. Both of the photometric redshift assignments are at redshifts sufficiently low that the silicate absorption would be accessible to the IRS spectral window, if present.

There is another interesting result regarding the eight sources for which there is evidence for photospheric emission in the IRAC bands. All sources except source 8 have a ratio of $f_{\nu}(24 \mu \mathrm{m}) /$ $f_{\nu}(8 \mu \mathrm{m})>9.5$, and these seven sources represent seven of the nine sources in the entire sample of 24 having $f_{\nu}(24 \mu \mathrm{m}) /$ $f_{\nu}(8 \mu \mathrm{m})>9.5$. This limiting ratio is that used by Yan et al. (2005) with the intention of locating starburst sources. While the SED fits are consistent with a substantial starburst component, we have already noted that only one of these sources (source 11) has an IRS spectrum that might conceivably show PAH emission.

\section{SUMMARY AND CONCLUSIONS}

We present IRS spectra and optical and infrared photometric data for 24 optically faint sources with $f_{\nu}(24 \mu \mathrm{m})>0.8 \mathrm{mJy}$ selected from within the $8.2 \mathrm{deg}^{2}$ Spitzer Boötes survey within the NOAO Deep Wide-Field Survey. The Boötes sample with IRS spectra comprises a set of 58 sources with high infrared to optical ratios $\left[\nu f_{\nu}(24 \mu \mathrm{m}) / \nu f_{\nu}(I)=\mathrm{IR} / \mathrm{opt}>50\right]$ and typical $f_{\nu}(24 \mu \mathrm{m})=$ $1 \mathrm{mJy}$. Redshifts have been previously determined for 34 of the 58 sources, with a median $z=2.2$. The 24 sources discussed in the present paper are the remaining sources that do not show sufficiently strong spectral features in the IRS low-resolution spectra for confident redshift determination by fitting with templates containing either strong silicate absorption or strong PAH emission.

There are no significant systematic differences in overall SEDs or fluxes for the sample with redshifts (usually having strong silicate absorption at rest frame $9.7 \mu \mathrm{m}$ ) and the sample without redshifts (having weak or no absorption or emission features). Of the 24 sources, 16 show power-law SEDs determined from photometry through infrared and optical wavelengths. Sources could be at $z \lesssim 2.8$ with weak spectral features, or could show no fea- tures in the spectrum because $z \gtrsim 2.8$ and features are redshifted out of the IRS spectral range. Ten of the 24 sources have $\log \left[f_{\nu}(24 \mu \mathrm{m}) / f_{\nu}(8 \mu \mathrm{m})\right]>1.0$, a value expected for starbursts, but none of the sources show PAH emission features in the infrared spectra normally associated with a luminous starburst.

Possible redshifts are suggested for 9 of the 24 sources based on fitting a profile of weak silicate absorption. Photometric redshift estimates are given for two sources whose SEDs show evidence of a stellar component exceeding the dust continuum, although neither of these sources shows PAH emission, and the photometric redshift does not agree with the weak silicate redshift for the one source with both measured. These suggested new redshifts provide evidence in favor of the explanation for the majority of the "no-z" sources that they are similar in nature and luminosity to the more heavily absorbed sources, but with weaker absorption features, and would bring the total number of redshifts determined for faint Boötes sources to 44 of the 58 sources observed, counting one photometric redshift. Even if the suggested new redshifts are correct, there remain 14 sources from the Boötes sample of 58 with no redshift estimates. With our present data, it is not possible to reach any conclusions regarding the nature of these remaining sources. Five of these 14 sources without redshifts are not detected at any optical wavelength in the NDWFS. Whether these represent a dusty population at redshifts higher than previously measured, or whether they represent a population of optically obscured sources at $z<2.8$ with weak spectral features remains ambiguous. Because such sources are a significant fraction of the optically faint sources in the Spitzer "1 mJy" population of $24 \mu \mathrm{m}$ sources, continued efforts to determine their nature are important.

We thank D. Devost, G. Sloan, and P. Hall for help in improving our IRS spectral analysis. We thank the staff of the Keck Observatory for their assistance with obtaining the $2.2 \mu \mathrm{m}$ observations. The W. M. Keck Observatory is operated as a scientific partnership between the California Institute of Technology, the University of California and NASA. It was made possible by the generous financial support of the W. M. Keck Foundation. We extend special thanks to those of Hawaiian ancestry on whose sacred mountain we are priveleged to be guests. Without their generous hospitality, none of the Keck observations presented herein would have been possible. We thank NOAO for supporting the NOAO Deep Wide-Field Survey; A. D. and B. J. acknowledge support from NOAO, which is operated by the Association of Universities for Research in Astronomy (AURA), Inc., under a cooperative agreement with the National Science Foundation. This work is based primarily on observations made with the Spitzer Space Telescope, which is operated by the Jet Propulsion Laboratory, California Institute of Technology under NASA contract 1407. Support for this work by the IRS GTO team at Cornell University was provided by NASA through Contract Number 1257184 issued by JPL/Caltech.
Alonso-Herrero, A., et al. 2006, ApJ, 640, 167

Brand, K., et al 2006, ApJ, 644, 143

Brown, M., et al. 2006, ApJ, 638, 88

Desai, V., et al. 2006, ApJ, 641, 133

Dey, A., et al. 2005, ApJ, 629, 654

Eisenhardt, P., et al. 2004, ApJS, 154, 48

Fazio, G., et al. 2004, ApJS, 154, 10

Genzel, R., et al. 1998, ApJ, 498, 579

\section{REFERENCES}

Higdon, S. J. U., et al. 2004, PASP, 116, 975

Houck, J. R., et al. 2004a, ApJS, 154, 18

2004b, ApJS, 154, 211

2005, ApJ, 622, L105 (H05)

Hunt, L., \& Maiolino, R. 2005, ApJ, 626, L15

Jannuzi, B. T., \& Dey, A. 1999, in ASP Conf. Ser. 191, Photometric Redshifts and the Detection of High Redshift Galaxies, ed. R. Weymann et al. (San Francisco: ASP), 111 
Li, A., \& Draine, B. 2001, ApJ, 554, 778

Martinez-Sanigre, A., Rawlings, S., Lacy, M., Fadda, D., Marleau, F. R., Simpson, C., Willott, C. J., \& Jarvis, M. J. 2005, Nature, 436, 666

Matthews, K., \& Soifer, B. T. 1994, Exp. Astron., 3, 77

Persson, S. E., Murphy, D. C., Krzeminski, W., Roth, M., \& Rieke, M. J. 1998, AJ, 116, 2475

Polletta, M., et al. 2006, ApJ, 642, 673

Rieke, G. H. 1976, ApJ, 210, L5

Rieke, G. H., et al. 2004, ApJS, 154, 25
Simpson, C., \& Eisenhardt, P. 1999, PASP, 111, 691

Smith, P. S., Schmidt, G. D., Allen, R. G., \& Angel, J. R. P. 1995, ApJ, 444, 146

Soifer, B. T., et al. 2004, ApJS, 154, 151

Spoon, H. W. W., et al. 2006, ApJ, 638, 759

Surace, J. A., \& Sanders, D. B. 2000, AJ, 120, 604

Thuan, T. X., Sauvage, M., \& Madden, S. 1999, ApJ, 516, 783

Weedman, D. W., et al. 2005, ApJ, 633, 706

Yan, L., et al. 2005, ApJ, 628, 604 